\title{
Measurement of wisdom forging force using piezoelectric materials
}

\author{
Shao-Yi Hsia ${ }^{1, *}, Y u-T u a n \mathrm{Chou}^{2}$, Chen-Chung Chang ${ }^{1}$, and Ling-Yu $\mathrm{Hsu}^{1}$ \\ ${ }^{1}$ Department of Mechanical \& Automation Engineering, Kao-Yuan University, Kaohsiung City, 821, Taiwan \\ ${ }^{2}$ Department of Applied Geoinformatics, Chia Nan University of Pharmacy \& Science, Tainan City, 717, Taiwan
}

\begin{abstract}
Aiming at forging machines for bolts and nuts with up to $61 \%$ market share, adding piezoelectric sensing units to the mold forging is discussed in this study. In the research process, it attempts to develop a sensing element with low cost, practicable limited space, acceptable performance stability, and real-time measurement of forging load by a five-stage forming system for special nuts matched with the theory of plastic forming, the $\mathrm{CAD} / \mathrm{CAE}$ design and numerical analysis, and the installation of a piezoelectric unit. It aims to assist fastener businesses in the intelligentization, networking, and systemization of machines and further integrate into the Internet service manufacturing system to enhance the overall competitiveness of fastener industry.
\end{abstract}

\section{Introduction}

Intelligent manufacturing is the inevitable product of mechanized, automated, and informatized application to the mature stage. The competitive elements of manufacturers therefore change from previous materials and energy to the resources of materials, energy, and information as the core competitiveness. Intelligent manufacturing refers to the establishment of digitized factories and intelligentized factories/manufacturers, through various intelligent manufacturing systems (or equipment) and automated or intelligentized production lines, to present multiple sensing devices for avoiding possible risks and reaching zero worry for enterprises $[1,2]$. Apparently, "intelligent manufacturing" or "Industry 4.0" stresses more on the application of automation intelligentized equipment, Internet of machines, Cyber-Physical System (CPS), cloud, and big data analysis. Nevertheless, fastener industry in Taiwan still exists in following technology gaps [1], when developing intelligent equipment.

(1) Sensing technology for molds, principal production equipment (e.g. forming machines, rolling machines), and sieve sorters has not been developed to real-time monitor the quality of fasteners in the process.

(2) M2M connection technology and communication protocol as well as the standards for equipment like forming machines have not been established.

(3) Technology for real-time mass data processing, analyzing, and predicting has not been established.

(4) There has not been a solution for production schedule CPS integration systems that the virtual and the reality could not be automatically integrated.

(5) There has not been a large manufacturer preceding the integration to provide a complete multidisciplinary service environment.
Finite Element Method could simultaneously analyze mechanical problems derived from all interface of blanks and molds under plastic deformation. Theoretically, there is almost no restriction to geometric complexity. The boom of technology in past years further accelerates the calculation with computers; besides, the development of simulation software has computer-assisted simulation technology become mature and stable. Finite Element Method therefore presents more superior use efficiency and accuracy. The application of Finite Element Method and the relevant technology to forging forming is reviewed as below.

O'Connell et al. [3] developed Slab method and Finite Element Method DEFORM-2D in 1996 to estimate the forming load of hexagon bolts trimless closed-die forging for selecting suitable forming machines, avoiding energy waste, and reducing the production speed. Hyunkee et al. [4], in 1996, designed the forming process sequence with a component by using FORMEX and Finite Element software DEFORM, to establish the program database for the process and effectively reduce the time for forming process sequence and mold design in the product production. MacCormack et al. [5] used Finite Element Analysis software DFFORM for the multiple-stage cold forging forming analysis in 2002 and observed the strain distribution, with three-stage damage and flow, after the product was formed in order to understand the failure value and the effective strain value. In the same year, Behrens et al. [6] utilized Finite Element Analysis software MSC.SuperForm 2002 for the failure analysis of various production processes and discussed the material breaks in cold forging and warm forging. The results revealed that the surface cracks could be observed under two situations and the material damage could be reduced by slightly modifying the geometric shape of the key workpiece.

Corresponding author: syhsia@cc.kyu.edu.tw 
$\mathrm{Su}$ [7] indicated in 2014 that the idea of sensors proposed by Rosenbla in 1957 became a primary part in the research on neural network. Moreover, Professor John Holland in University of Michigan, in order to develop artificial intelligence systems, developed Genetic Algorithm in 1960 by referring to the solution for the mathematical optimization.

Hsia et al. [8], in 2016, attempted to experiment multiple-stage fastener cold forging forming machines with accelerometers and vibration testing software by capturing the background vibration at the feeding system and starting the feeding system but not feeding materials as well as the vibration signals of time for the feeding system starting to feed materials. MTALAB software was further used for the FFT analysis to view the distribution of frequency spectra.

Although there have been several researchers proposing various studies on fastener forging forming to solve the practical problems on sites, the introduction of sensing technology to the promotion of intelligent manufacturing, attempting to proceed real-time monitoring and diagnosis technology on fastener molds and principal production equipment (e.g. forming machines, rolling machines) matched with sensing units, is still at the beginning stage. In this study, the theory of plastic forming is matched with the $\mathrm{CAD} / \mathrm{CAE}$ design and numerical analysis to design and develop a piezoelectric measurement unit, expecting to real-time measure the forging load to assist fastener businesses in promoting intelligentized manufacturing and to further enhance the overall competitiveness of the industry.

\section{Basic principle}

\subsection{Cold forging forming}

Metal forming is an extremely complicated deformation process, on which material characteristics, temperature conditions, friction conditions, lubrication, blank size, and mold shape present certain degree of influence. During theoretical analyses, people could merely change difficult mechanical problems in mathematics into engineering problems through some hypotheses and experimental or empirical data because of material nonlinearity (nonlinear relationship between stress and strain) and geometric nonlinearity (nonlinear relationship between strain and displacement) as well as the complexity of boundary conditions and the difficulty in mathematics. Various analyses or mathematical models with different approximation and application are therefore generated, including principal stress method, slip line method, upper bound method, lower bound method, and Finite Element Method. With Finite Element Method, materials are divided into several elements (triangular, quadrilateral) to calculate the strain and stress of elements with multiple simultaneous equations so as to be analyzed with computers and applied to differnt field in past years. Finite Element forming software DEFORM 3D is utilized in this study for the simulation and analysis.

\subsection{Piezomaterial unit}

In addition to the dielectric properties and elastic behavior of general materials, piezoelectric ceramics also present piezoelectric properties. Numerous performance parameters are the primary basis of piezoelectric ceramics being broadly applied.

\section{(1) Piezoelectric constant}

Piezomaterial presents both mechanical and electrical characteristics that the constitutive law is denoted as below.

$$
\begin{aligned}
& \sigma_{\mathrm{ij}}=C_{\mathrm{ijkl}}^{\mathrm{E}} \varepsilon_{\mathrm{kl}}-e_{\mathrm{mij}} E_{\mathrm{m}} \\
& D_{\mathrm{n}, \mathrm{n}}=e_{\mathrm{mkl}} \varepsilon_{k l}+\mu_{m n}^{\sigma} E_{\mathrm{m}}
\end{aligned}
$$

where $\sigma_{\mathrm{ij}}$ : stress tensor

$$
\varepsilon_{k l}: \text { strain tensor }
$$

$E_{\mathrm{m}}$ : electric field vector

$C_{\mathrm{ijkl}}^{\mathrm{E}}:$ stiffness tensor of constant electric field

$e_{\mathrm{mkl}}$ : piezoelectric tensor

$\mu_{m n}^{\sigma}$ : permittance tensor of constant elastic stress

The mechanical characteristics should conform to the equation of motion of an elastomer.

$$
\sigma_{\mathrm{ij}, \mathrm{j}}+f \mathrm{i}=\rho \ddot{u}_{\mathrm{i}}
$$

Meanwhile, when the linear elastic behavior of small deformation is taken into account, the relationship between displacement and strain is denoted as below.

$$
\varepsilon_{i j}=\frac{1}{2}\left(u_{\mathrm{i}, \mathrm{j}}+u_{\mathrm{j}, \mathrm{i}}\right)
$$

Regarding the electrical characteristics, it is assumed that the magnetic effect caused by electric fields is extremely small that it could be ignored. Meanwhile, the electric charge in piezomaterial is little that it could be ignored. The Maxwell electromagnetic equation therefore could be simplified as

$$
D_{\mathrm{n}, \mathrm{n}}=0
$$

In real situations, the measured and applied physical quantity is voltage that the electric field is transformed into voltage.

$$
E \mathrm{i}=-\phi_{\text {, }}
$$

$\phi$ : electric potential

(2) Electromechanical coupling coefficient

The electromechanical coupling coefficient $k$ is a parameter comprehensively reflecting piezomaterial properties, presenting the coupling effect between the mechanical energy and the electrical energy of piezomaterial. The electromechanical coupling coefficient could be defined as

$k^{2}=\frac{\text { Electrical energy converted into mechanical energy }}{\text { Input electrical energy }}$

or

$k^{2}=\frac{\text { Mechanical energy converted into electrical energy }}{\text { Input mechanical energy }}$

\section{Research method and architecture}

\subsection{Research method}

The forging force required for fasteners generally relies on the experience of manufacturers. For instance, it requires about five stages to gradually complete a special nut diagonal of about $11.33 \mathrm{~mm}$. A five-mold forging 
machine, LS-C-NF-14B-5F, of a company could forge the nut diagonal below $14 \mathrm{~mm}$, and the machine could bear the load about $850 \mathrm{kN}$. However, no-one knows whether the amount is just enough or would have allowance. Matching the simulation of forming software could acquire the forging force at each stage; nonetheless, measuring with a commercial load cell would be restricted to the exceeding forging force at a single stage. In this case, a larger size load cell is necessary. Nevertheless, merely a certain size load cell could be placed between molds at each stage because of the limited space in the forging machine.

Accordingly, the plastic forming analysis is combined with a piezoelectric sensing unit in this study to develop a sensor which is suitable for the fastener forming site. The developed sensing unit presents the following advantages.

(1) The expense for the developed piezoelectric sensor is far lower than imported or domestically developed load cells or signal sensors.

(2) The modified total forging force analysis model could assist in the selection of forging machine specifications for innovative fasteners and avoid over investment or overload operation. For an equipment manufacturer, it could accurately quote the price and enhance the product price-performance ratio. For a fastener manufacturer, on the other hand, it could correctly acquire the forging force to accurately control the production process and make failure diagnoses and exclusion in advance.

(3) The forging force waveform could be correctly monitored to judge the degradation of molds. When ISO2372 standards are matched, a mold could be replaced at proper time.

AISI-1010 materials are selected for the experiment in this study. Five-stage special nuts, Figure 1, which requires five stages for the completion step by step, are the research target. SolidWorks is matched to construct the geometric design, and Finite Element forming software is introduced for the simulation to acquire the forging force at each stage. The research process is shown in Figure 2. SolidWorks is matched in the beginning to establish the geometric figure, and then the forming software is used for the simulation and analysis, which is further verified with the measuring results.

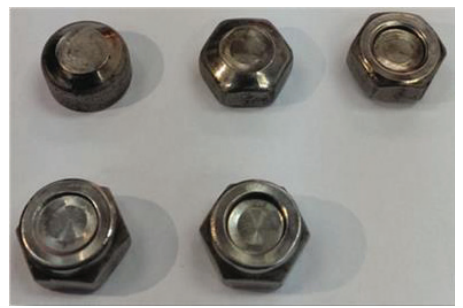

Fig. 1. Cold forging of five-stage special nuts.

\subsection{Steps}

(1) Introduction of machine and fastener

Special nuts with simpler geometric shape (Figure 1) are analyzed in this study. The product is preceded from wire cutting, material arranging, and preforming to the final finished product through five-mold cold forging (Figure 3, five-stage nut forging machine), and the forging force at each stage is acquired with simulation.

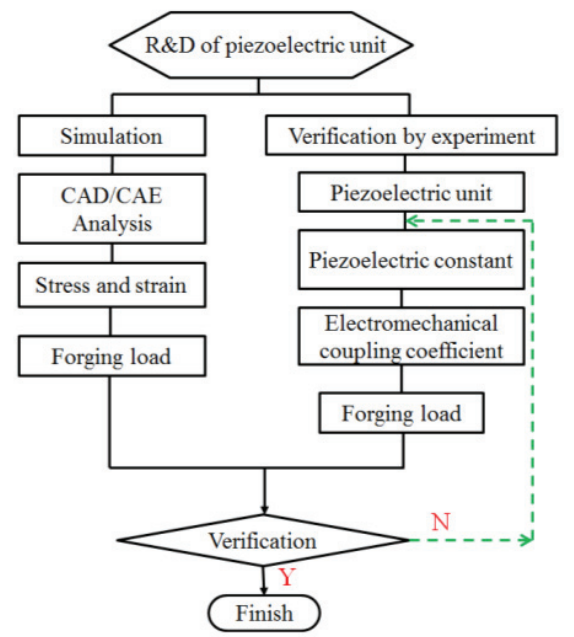

Fig. 2. Flow chart of this study.

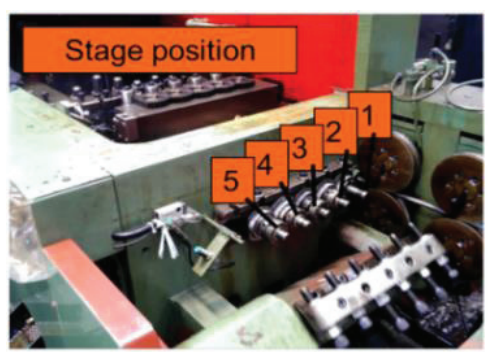

Fig. 3. Five-stage nut forging machine.

(2) Manufacturing and use of piezoelectric unit

Current piezoelectric sensors embedded in most forging machines could merely be used for the comparison with continuous signals, but do not show absolute relationship with forging force. General piezoelectric units, because of low prices, could not provide the sensitivity $(\mathrm{mv} / \mathrm{g})$ of common acceleration probes or the conversion coefficient $(\mathrm{mv} / \mathrm{N})$ of load cells, and the piezoelectric unit properties can hardly maintain the approximate stability. For this reason, it is important to acquire conversion coefficients and maintain certain performance curve. The piezoelectric unit utilized in this study, Figure 4, is embedded at the second to the fifth stages, Figure 5.

(3) Application of $\mathrm{CAD} / \mathrm{CAE}$ forming design and analysis software

Before the cold forging forming simulation, the mechanical properties of materials could be analyzed to conform to the actual production conditions, and then the cylindrical test is preceded and analyzed the results, e.g. flow stress. SolidWorks, the computer-assisted design software, is further utilized for drawing the production molds on site, including punch pin, workpiece, die, and punch, and transforming into STL files for the simulation with Finite Element forming analysis software DEFORM. Convergence Analysis is used in the research process for acquiring proper number of grids to remain the forming stability as well as shorten the time for computer simulation and calculation. 


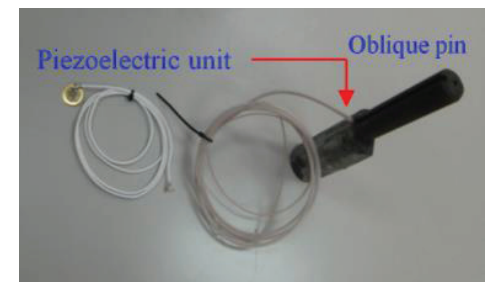

Fig. 4. Piezoelectric unit embedded in the oblique pin.

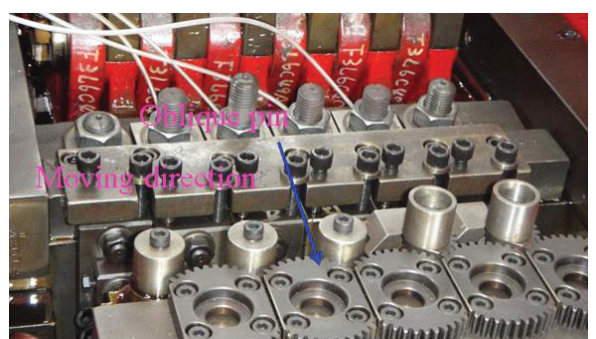

Fig. 5. Four sets piezoelectric unit embedded at the second to the fifth stages.

\section{Results and discussion}

Figure 6 shows the effective stress distribution from the first to the fifth stages, where the first and the second stages are the preforming that the born stress is smaller; the fourth and the fifth stages are the principal forming stage. Figure 7 displays the effective strain distribution from the first to the fifth stages, where the strain mostly appears on the fourth and the fifth stages and concentrates on the central deformation area.
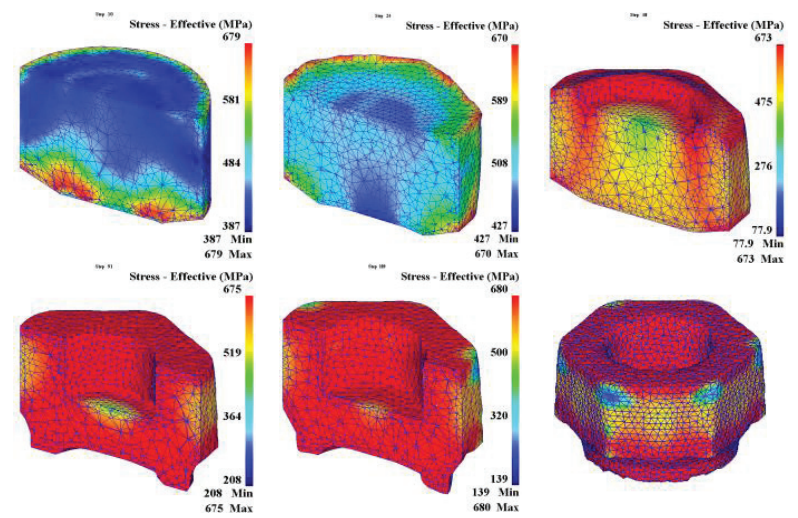

Fig. 6. Effective stress distribution from the first to the fifth stages and full scale.
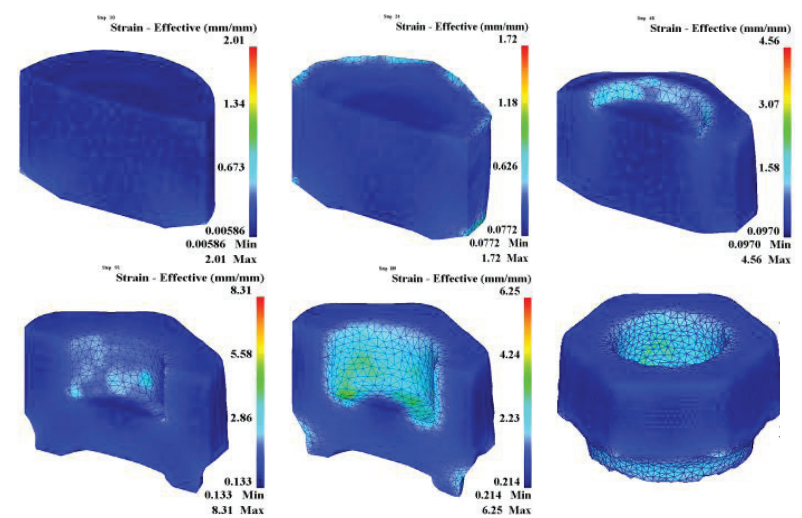

Fig. 7. Effective strain distribution from the first to the fifth stages and full scale.
This section analyzes the forging load from the first stage to the fifth stage, as displayed in Fig. 8. In the preforming of the workpiece in the first stage, the forging load is $121 \mathrm{kN}$ around $14.37 \%$ of the total punch loads in the five stages. In forming in the second stage, the forging load is $185 \mathrm{kN}$ approximately $21.97 \%$. In the third stage, the forging load, $188 \mathrm{kN}$ approximately $22.32 \%$, is larger than in any other stage. In the fourth stage, the forging load, $185 \mathrm{kN}$ around $21.97 \%$, has the same value as the second stage in this study. Overall, the total forging load for forming special nut from the first stage to the fourth stage was $842 \mathrm{kN}$.

Figure 9 and Figure 10 present the forging load at the third and fifth stages measured by the embedded piezoelectric unit, where (a) is the simulation results and (b) is the magnification of a single signal acquired from the measurement. By comparing (a) and (b), the simulation results and the measured signals exist in certain relationship. When the piezoelectric constant of the piezoelectric unit and the electromechanical conversion coefficient could be studied and discussed in the future, the relationship between electrical signals and forging force would be discovered to assist fastener businesses in the machine intelligentization, networking, and systemization as well as to enhance the overall competitiveness of the fastener industry.

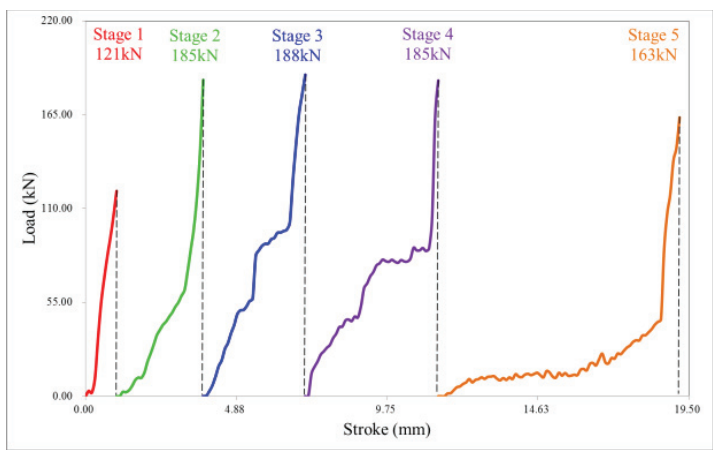

Fig. 8. Forging load from the first to the fifth stages with simulation method.

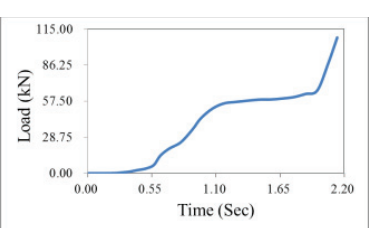

(a)

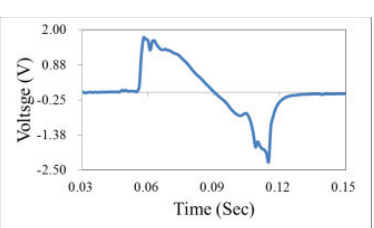

(b)
Fig. 9. Forging load at the third stage measured by the embedded piezoelectric unit: (a) simulation results, (b) magnification of a single signal.

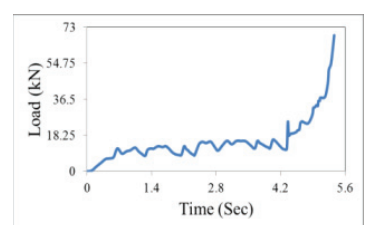

(a)

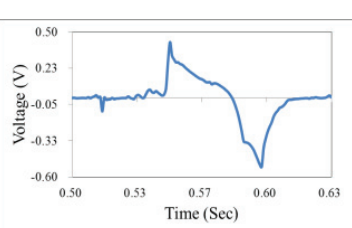

(b)
Fig. 10. Forging load at the fifth stage measured by the embedded piezoelectric unit: (a) simulation results, (b) magnification of a single signal. 


\section{Conclusions}

This study could assist businesses in verifying the accuracy of the software analysis by the acquisition of forging force. In the future, the development of any newstyle molds could be analyzed and modified with the forming software before contracting for manufacturing so as to achieve the best economic benefits. The relevant conclusions are summarized as following.

(1) Finite Element Analysis software DEFORM-3D matched with experimental data for the simulation and analysis could shorten the time for mold testing to reduce human expenses and production costs as well as save materials and reduce equipment loss.

(2) Using the piezoelectric sensing unit for measuring forging force could assist fastener businesses in the machine intelligentization, when acquiring the research data like piezoelectric conversion conefficients, so that "the intelligent manufacturing system with introspective prediction could avoid risks in advance and present zero worry for enterprises."

\section{References}

1. C.Y. Chen, C.C. Yeh, Application of intelligent manufacturing to fastener industry, ITIS research topic, Metal Industries Research \& Development Centre, (2016)

2. H.-Y. Chi, Outlook and review of 2015 fastener industry in Taiwan. Industry Report, 82-84 (2016)

3. M. O'Connell, B. Painter, G. Maul, T. Altan, Flashless closed-die upset forging-load estimation for optimal cold header selection, Journal of Materials Processing Technology, 59(1-2), 81-94 (1996)

4. H. Kim, T. Altan, Cold forging of steel-practical examples of computerized part and process design, Journal of Materials Processing Technology, 59, 122-131 (1996)

5. C. Maccormack and J. Monaghan, 2D and 3D finite element analysis of a three stage forging sequence, Journal of Materials Processing Technology, 127, 48-56 (2002)

6. A. Behrens and H. Just, Extension of the forming limits in cold and warm forging by the FE based fracture analysis with the integrated damage model of effective stresses, Journal of Materials Processing Technology, 125-126, 235-241 (2002).

7. S.Su, Shape Optimization design of cold forging punch, (2014)

8. S.Y. Hsia, Y.C. Li, H.-H. Hsu, Y.T. Chou, and C.C. Yang, Vibration analysis of multiple-stage fastener cold forging forming machine feeding system, 2016 TSTP Taiwan Society for Technology of Plasticity, No. B2-6(29) (2016)

9. Ultrasonic flaw detection editors, Ultrasonic flaw detection, Industry Report, Hydroelectric power press, 75-90 (1980)

10. H.S. Huang, S.Y. Hsia, and S.K. Yang, 2016, Optimal design of ultrasonic transducer system applied on biolipid extraction from Chlorella, Sensors and Materials, 28(5), 511-522 (2016)

11. K. Nakamura, Ultrasonic transducers-Materials and design for sensors, actuators and medical applications, Woodlead publishing (2012) 